\title{
Molecular characterization of a new urea transporter in the human kidney
}

\author{
Bernadette Olivès $^{\mathrm{a}}$, Sonia Martial ${ }^{\mathrm{b}}$, Marie-Geneviève Mattei ${ }^{\mathrm{c}}$, Giorgio Matassi ${ }^{\mathrm{a}}$, \\ Germain Rousselet $^{\mathrm{b}}$, Pierre Ripoche ${ }^{\mathrm{b}}$, Jean-Pierre Cartron ${ }^{\mathrm{a}, *}$, Pascal Bailly ${ }^{\mathrm{a}}$ \\ ${ }^{a}$ INSERM U76, GIP-Institut National de la Transfusion Sanguine, 6 rue Alexandre Cabanel, 75015 Paris, France \\ ${ }^{\mathrm{b}}$ Service de Biologie Cellulaire, C.E.A./Saclay, 91191 Gif-sur-Yvette, France \\ 'INSERM U242, Hôpital de la Timone, 13385 Marseille, France
}

Received 14 March 1996; revised version received 11 April 1996

\begin{abstract}
A cDNA clone (HUT2) sharing $61.1 \%$ and $89.9 \%$ sequence identity with the human erythroid (HUT11) and the rabbit (UT2) urea transporters, respectively, was isolated by homology cloning from a human kidney library. HUT2 transcripts were restricted to the kidney and the HUT2 polypeptide was not immunoprecipitated with blood group Kidd-related antibodies (anti-Jk3) in coupled transcriptiontranslation assays. Functional expression studies in Xenopus oocytes demonstrated that HUT2-mediated urea transport was not inhibited by $p$-chloromercuribenzene sulfonate (pCMBS) which, however, inhibited the urea flux mediated by HUT11. These findings demonstrate that at least two distinct urea transporters are present in human tissues. By in situ hybridization, the gene encoding HUT2 has been assigned to chromosome 18q12.1-q21-1, as found previously for the Kidd/urea transporter HUT11, suggesting that both genes evolved from duplication of a common ancestor.
\end{abstract}

Key words: Urea transport; Human kidney; Tissue expression; Transcription-translation assay; In situ hybridization

\section{Introduction}

In mammalian cells, urea is the chief end-product of nitrogen catabolism and plays an important role in the urinary concentration mechanism $[1,2]$. Thus, the plasma membrane of human erythrocytes [3,4] and some renal epithelial cells [5] exhibit an elevated urea permeability which is mediated by highly selective urea transporters. At least two forms of urea transporters have recently been identified: (i) the presumably vasopressin-sensitive rabbit and rat transporters UT2 [6,7] and (ii) the constitutive human transporter HUT11 [8].

As expected for a vasopressin-regulated transporter, UT2 is localized in the inner medullary collecting ducts and probably in the descending thin limbs of Henle's loop [9], where it plays a central role in the establishment of a corticomedullary osmotic gradient. Two transcripts $(4.0$ and $2.9 \mathrm{~kb})$ of a rat urea transporter (rUT2) were identified in the kidney [7]. They have a spatially distinct distribution and their expression is modulated by different pathways. Indeed, the $4.0 \mathrm{~kb}$ transcript is confined to the inner medulla and is responsive to changes in the protein diet, whereas the $2.9 \mathrm{~kb}$ transcript is predominantly expressed in the inner stripe of the outer medulla and is responsive to the hydration state [7]. Thus, the two rUT2 messages are independently regulated by as yet unknown fac-

\footnotetext{
*Corresponding author. Fax: (33) (1) 47347431.
}

tors, critical for the physiological and functional status of the kidney.

The human urea transporter (HUT11 clone) has been cloned by cross-hybridization from a human bone marrow library [8]. The predicted translation product is a protein of 391 amino acids that displays $62.4 \%$ amino acid identity and a similar membrane topology to the rabbit transporter. HUT11 is responsible for the facilitated urea transport in human red cells and the mRNA is present in hematopoietic cell lines and in human (tumoral) kidney. It has been demonstrated that HUT11 is the product of the Kidd blood group locus [10] and that the rare Jk(a-b-) red cells lack the Kidd/urea transport protein showing a selective defect of the urea transport capacity $[10,11]$ whereas water permeability and aquaporinassociated Colton blood group antigens are normally expressed [10]. Recently, immunofluorescence analysis of human kidney sections demonstrated that the Kidd/urea transporter protein HUT11 is constitutively expressed by endothelial cells of vasa recta in the inner and outer medulla kidney, but not in renal tubules [12]. This is in agreement with data indicating that the descending vasa recta contains a urea transporter $[13,14]$.

Other urea transporters are likely to exist in tissues that use facilitated urea transport to manage the nitrogen catabolism [15]. Here we isolated the human homologue of the vasopressin-regulated rabbit UT2 clone by homology cloning from a human kidney library. The functional activity, immunochemical specificity, expression pattern and chromosome localization of the human transporters have been compared.

\section{Materials and methods}

\subsection{Library screening}

The UT2 cDNA encoding the rabbit urea transporter [6] was used as a random primed $\left[{ }^{32} \mathrm{P}\right] \mathrm{dCTP}$-labeled probe to screen about $1.7 \times 10^{6}$ bacteriophage clones of a human kidney cDNA library $5^{\prime}$ stretch purchased from Clontech laboratories (Palo Alto, CA). Positive clones were isolated and enzymatically amplified (annealing temperature $60^{\circ} \mathrm{C} ; 30$ cycles) between $\lambda$ gt 11 primers. To prevent PCR errors, we employed the Pwo DNA polymerase (Boehringer, Mannheim, Germany), which exhibits proofreading activity, and we sequenced 3 independent clones. All PCR products were ligated into the SmaI-digested pUC vector and nucleotide sequences were determined on both strands by the dideoxy chain termination method (Sanger) using the Sequenase enzyme (US Biochemical Corp).

\subsection{In situ hybridization}

The $730 \mathrm{bp} \mathrm{NcoI}$ fragment from the HUT2 cDNA clone was subcloned into pUC18 and ${ }^{3} \mathrm{H}$-labeled by nick-translation to a specific activity $8 \times 10^{7} \mathrm{dpm} / \mu \mathrm{g}$. Hybridization to metaphase chromosome, Giemsa staining after R-banding and autoradiography were performed as previously described [16]. 


\subsection{Northern blot analysis}

Poly $\left(\mathrm{A}^{+}\right)$RNAs ( $2 \mu \mathrm{g}$, Clontech) from adults and fetuses (pool of different gestational ages) per lane were size separated on a denaturing formaldehyde $1.2 \%$ agarose gel, transferred to nylon membrane and fixed by UV light. Absence of degradation was checked by staining with ethidium bromide. The Northern blots were hybridized at $42^{\circ} \mathrm{C}$ in the presence of $50 \%$ formamide with the ${ }^{32} \mathrm{P}$-labeled probes corresponding to the $N c o$ I fragment $(730 \mathrm{bp}$ ) of the coding region of HUT2 and to the full length HUT11 cDNA clone. Stringent washes were performed $\left(0.1 \times \mathrm{SSC}, 0.1 \% \mathrm{SDS}\right.$ at $\left.42^{\circ} \mathrm{C}\right)$ according to the guidelines of the manufacturer.

\subsection{Transcription-translation and immunoprecipitation assays}

The HUT2 and HUT11 cDNAs were subcloned into the EcoRVdigested pT7TS plasmid (kindly provided by P. Krieg, Austin, TX) under the control of the $\mathrm{T} 7$ promoter. The related proteins were synthesized in vitro using the transcription-translation coupled reticulocyte lysate kit from Promega (Madison, WI) in the presence of $\mathrm{L} .\left[{ }^{35}\right.$ S $]$ methionine (Amersham, Bucks, UK) according to the manufacturer's protocol. These reaction mixtures were used in immunoprecipitation assay with the human anti-Jk ${ }^{3}$ antiserum as previously described [10]. The different products obtained were analyzed by SDSPAGE (15\% separating gel) on a discontinuous buffer system [17] and followed by autoradiography.

\subsection{Oocyte urea flux measurements}

After linearization of the pT7TS-HUT11 and -HUT2 construct vectors with $S m a \mathrm{I}$ and $E c o$ RI restriction enzymes, respectively, capped sense RNAs were synthesized using T7 RNA polymerase from the mCAP mRNA capping kit (Stratagene, La Jolla, CA). Expression studies were carried out by microinjection of cRNAs (10 ng/ oocyte) in collagenase-treated Xenopus laevis oocytes [18] and functional tests were realized 2 or 3 days after injection as described previously [8].

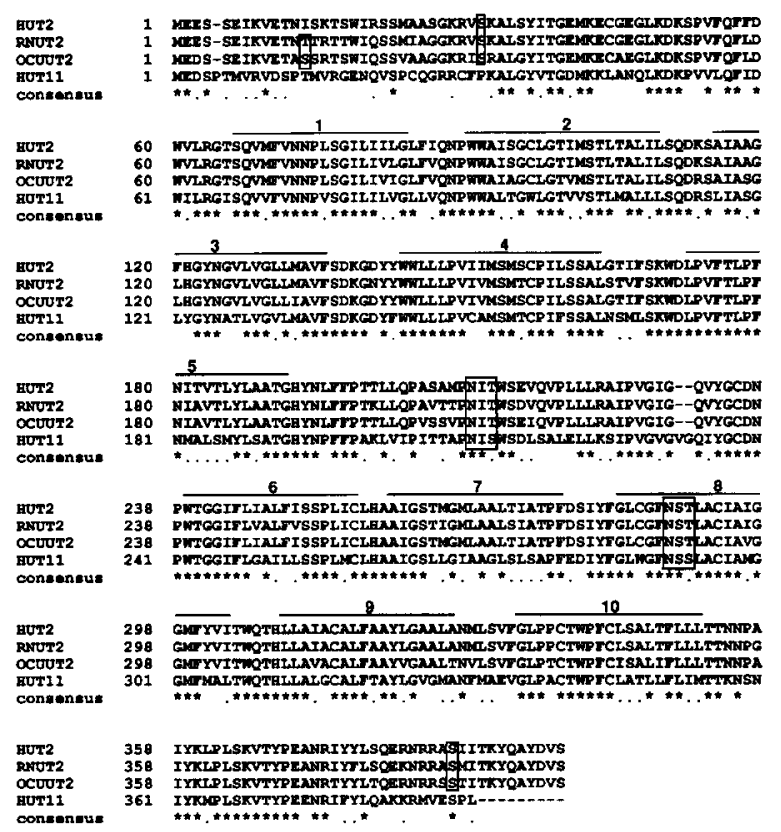

Fig. 1. Multiple sequence alignment of urea transporters. Amino acid sequence alignment (CLUSTAL W program [19]) of HUT2 with the rat (RNUT2), rabbit (OCUUT2) and human Kidd/urea transporter protein HUT11. Conserved residues and conservative types are indicated as stars and as dots, respectively. Gaps appear as dashes. The 10 predicted membrane-spanning regions of rat UT2 protein are numbered and indicated by a solid line. Potential $\mathrm{N}$-glycosylation and phosphorylation sites are boxed. HUT2 nucleotide sequence deposited to the EMBL data base (accession number X96969).

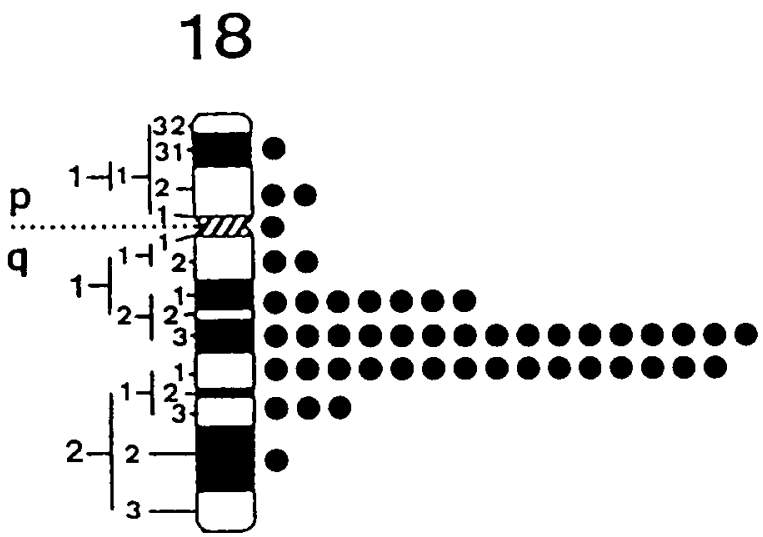

Fig. 2. Chromosome localization of HUT2. Histogram of the human G-banded chromosome 18, illustrating the distribution of labeled sites with the HUT2 probe. In the 100 metaphase cells examined, 204 silver grains associated with chromosomes, among which $23.5 \%$ were located on the long arm of chromosome 18 . Over $79.2 \%$ mapped to the $18 \mathrm{q} 12.1-\mathrm{q} 21.1$ region.

\section{Results and discussion}

\subsection{Cloning of a new human kidney urea transporter}

The HUT2 clone was isolated and characterized from the human kidney library by homology cloning with the rabbit UT2 cDNA probe. Alignment of the deduced amino acid sequence with those of the other known urea transporters is shown in Fig. 1.

The HUT2 clone encoded a polypeptide chain of 397 amino acids that exhibited $92.3 \%$ and $89.9 \%$ similarity with the rat and rabbit UT2 proteins, respectively, but only $61.1 \%$ similarity with the urea transporter of human erythrocytes HUT11. Comparison of UT2 and HUT2 indicated that two potential phosphorylation sites (for protein kinase A), which are lacking in HUT11, the two N-linked glycosylation sites and the 10 cysteines were conserved at the same positions (Fig. 1). However, the potential protein kinase $\mathrm{C}$ phosphorylation site conserved in the rat and rabbit UT2, is lacking in HUT2.

Another difference between the HUT2, rat and rabbit transporters on the one hand and the HUT11 transporter on the other hand, concerned the base composition of their nucleotide coding sequences. The $\mathrm{G}+\mathrm{C}$ content at synonymous variable codon positions was much higher in the first group of genes $(69 \%, 71 \%$ and $74 \%$ respectively) than in the second $(56 \%)$. In mammals, the $\mathrm{G}+\mathrm{C}$ content at silent sites (codon usage) correlates with the GC level of the genomic region (isochore) surrounding each gene (for reviews see $[20,21]$ ). Therefore, weakly constrained sites (i.e. synonymous sites) in coding sequences may have evolved under the same mutational pressure affecting the region where the gene was embedded [22]. This evolutionary model seems to apply to the two types of human urea transporters analyzed in this work. Gene duplication may have been followed by divergence taking place in two different genomic environments. Thus, amino acid sequence and base composition comparisons suggest that HUT2 is the human homolog of the rabbit or rat UT2 transporters.

\subsection{Chromosomal localization}

The gene encoding HUT2 was localized on human chromo- 

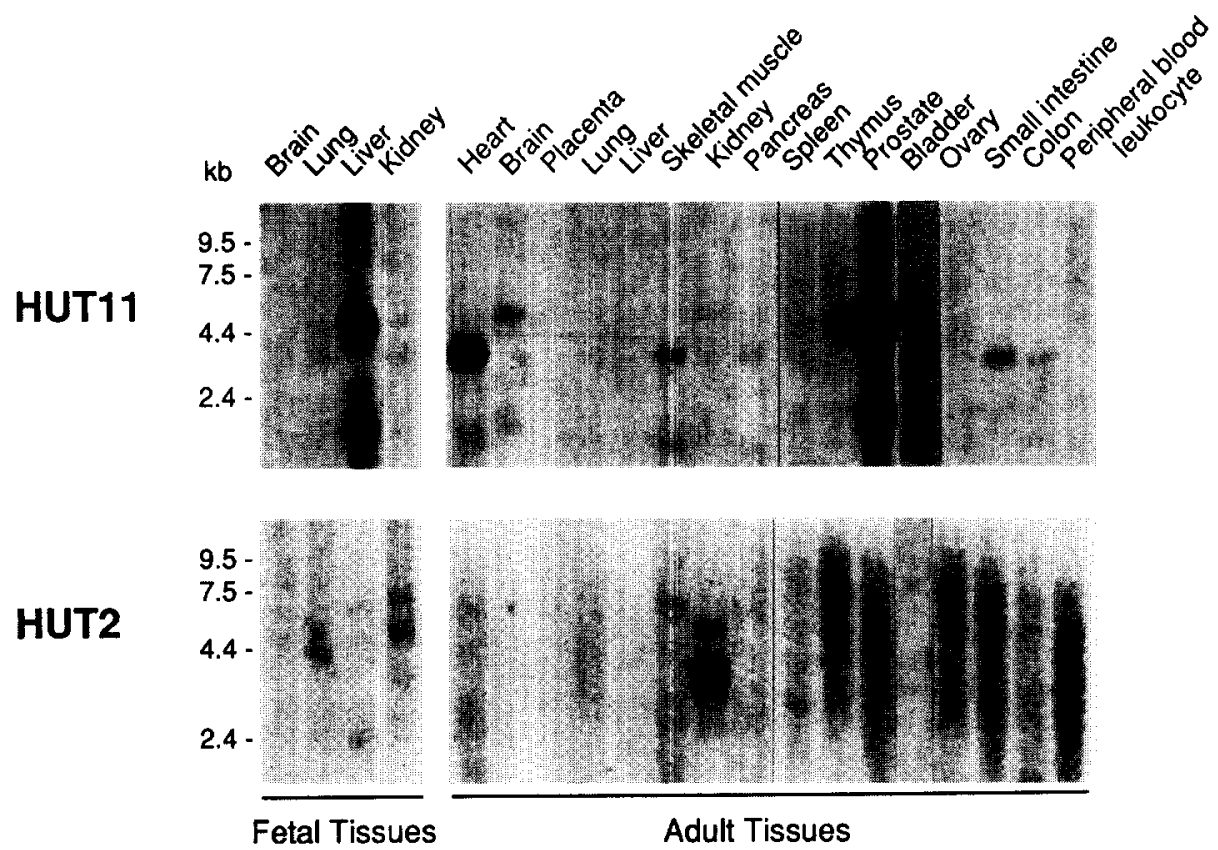

Fig. 3. Northern analysis of HUT11 and HUT2 expression in human tissues. Poly $\left(\mathrm{A}^{+}\right)$RNA $(2 \mu \mathrm{g})$ from indicated human tissues (adult and fetal) were hybridized with ${ }^{32}$ P-labeled HUT11 and HUT2 cDNA probes as described in Section 2. Size markers (kb) are indicated on the left.

somes by in situ hybridization with the NcoI cDNA fragment. This probe mapped to the q12.1-q21.1 region of chromosome 18 (Fig. 2), a position identical to the HUT11/Kidd blood group locus previously identified [10,23]. These results further suggest that both genes evolved from duplication, after that the internal repeats (found within HUT11 and UT2 sequences), most likely resulting from another duplication, has occurred [24].

\subsection{Tissue distribution of HUT11 and HUT2 mRNAs}

Northern analysis revealed that HUT11 was predominantly expressed in human fetal liver, adult prostate and bladder as transcripts of 4.5 and $2.0 \mathrm{~kb}$ (Fig. 3). A similar pattern was previously detected in spleen erythroblasts [8]. HUT11 transcripts were also detected in other organs with distinct patterns. A weak signal at $4.5 \mathrm{~kb}$ was detected in thymus and brain. In heart, skeletal muscle, colon and small intestine, a predominant signal was detected at $3.6 \mathrm{~kb}$ with a weak band at $2.0 \mathrm{~kb}$. A similar pattern was also observed in pancreas. In the adult kidney, there was only a very faint signal, consistent with the immunochemical localization of this transporter in endothelial cells of the vasa recta, but not in renal tubules [12]. This is also consistent with the detection of the transcripts in hydronephrotic rat kidneys [25].

When total RNAs from adult human kidney were analysed with the HUT2 cDNA, a faint signal was found at $4.5 \mathrm{~kb}$ and a strong signal at $3.6 \mathrm{~kb}$. Preliminary studies by in situ hybridization on kidney sections revealed that HUT2 was selectively expressed by inner medulla collecting ducts, a nephron segment known to be under the regulatory control of vasopressin (E. Rondeau, P. Bailly and J.P. Cartron, unpublished data). In the fetal kidney only very weak hybridization signals (possibly cross-hybridization) were detected on Northern blots (Fig. 3), suggesting up-regulation during development. No clear signal could be detected in the other tissues examined (Fig. 3). Interestingly, the HUT2 transcript was not (or only very weakly) detected in the human colon (Fig. 3), at the opposite of the UT2 transcript which was present in the rabbit colon [6]. Recently, it was reported that the rat UT2 transcript was undetectable in the colon and it was suggested that this may represent an adaptation towards the dietary protein consumed between herbivore (rabbit) and omnivore (rat) [7].

Taken together, our results indicate that HUT11 is present in several tissues whereas HUT2 is restricted to the kidney. HUT11 might be constitutively expressed and involved in the transport of urea in many organs whereas the HUT2 transporter might be vasopressin-inducible and restricted to the kidney to participate in the urinary concentration mechanism.

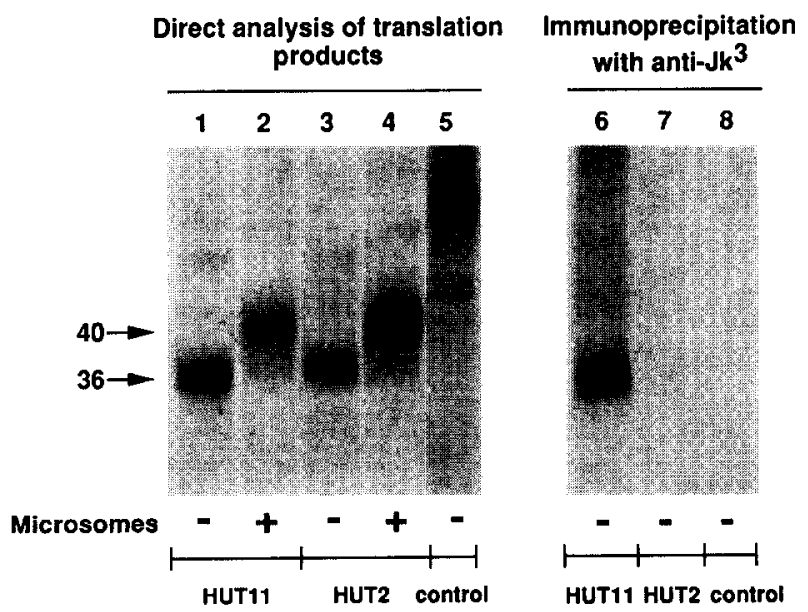

Fig. 4. Expression of urea transporters in transcription-translation assays. Left, autoradiogram of $\mathrm{L}-\left[{ }^{35} \mathrm{~S}\right]$ methionine-labeled proteins analyzed by SDS-PAGE (15\% gels). Lanes 1 and 2: pT7TS-HUT11 with and without canine pancreatic microsomal membranes; lane 3 and 4: pT7TS-HUT2 with and without microsomes; lane 5: control luciferase plasmid. Right: autoradiogram of ${ }^{35} \mathrm{~S}$-labeled proteins immunoprecipitated with a human anti-Jk ${ }^{3}$ and separated by SDSPAGE. Lanes 6 and 7: immunoprecipitates from pT7TS-HUT11 and pT7TS-HUT2, respectively; lane 8: luciferase control. Arrows on the left refer to product size $(\mathrm{kDa})$. 
Table 1

Urea permeabilities of water, HUT11 and HUT2 injected oocytes

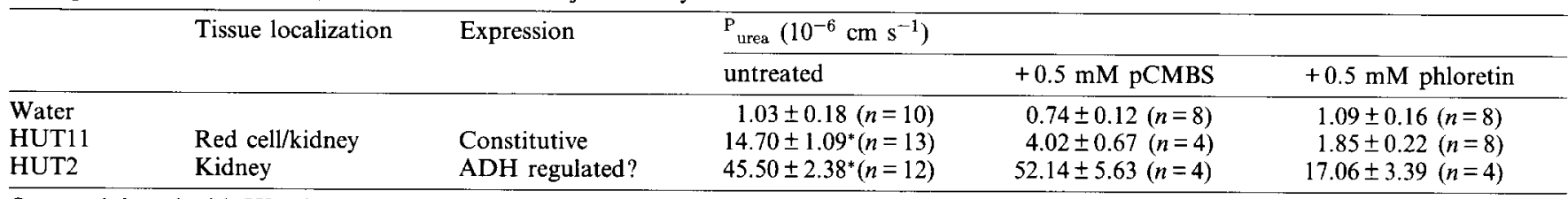

Oocytes injected with HUT2 cRNAs were incubated individually in $200 \mu \mathrm{l}$ of Barth's medium containing $8 \mu \mathrm{Ci}(0.145 \mathrm{mM})\left[^{14} \mathrm{C}\right] \mathrm{urea}(\mathrm{Amersham}$, $\mathrm{UK}$ ) and $5 \mu \mathrm{Ci} / \mathrm{ml}\left[{ }^{3} \mathrm{H}\right]$ raffinose (Dupont NEN, Germany) as a control of the oocyte integrity. Oocytes injected with HUT11 and water were used as positive and negative controls. Incubation time was $90 \mathrm{~s}$ at $18^{\circ} \mathrm{C}$ under gentle shaking. After washing and solubilization, the samples were counted by liquid scintillation in a Tri-Carb 4000 scintillation counter (Packard Inst., Rungis, France). For inhibition experiments, 0.5 mM phloretin (Sigma) and $0.5 \mathrm{mM}$ para-chloromercuribenzene sulfonate (pCMBS) (Sigma) were added, 20 min and 10 min, respectively, before the assay and maintained during the urea uptake. Urea permeability was calculated from the oocyte-associated amount of $\left[{ }^{14} \mathrm{C}\right]$ urea and the oocyte surface area was calculated from the optically determined apparent diameter. Data are presented as mean \pm S.E. $(n)$, where $n$ indicates the number of oocytes for each experimental condition.

${ }^{*} P<0.001$ vs. water-injected oocytes; $P<0.001$ vs. untreated oocytes.

\subsection{Coupled transcription-translation of HUT11 and HUT2 cDNAs}

In the transcription translation coupled reticulocyte lysate system, both plasmids pT7TS-HUT11 and -HUT2 directed the synthesis of 36 and $40 \mathrm{kDa}$ protein bands when carried out with and without canine pancreatic microsomal membranes, respectively (Fig. 4, lanes 1-4). The translated product from HUT11, but not from HUT2, could be immunoprecipitated with the anti- $\mathrm{Jk}^{3}$ antibody, produced by immunized $\mathrm{Jk}(\mathrm{a}-\mathrm{b}-$ ) individuals (Fig. 4, lanes 6-8). Thus, the HUT2 polypeptide does not carry the $\mathrm{Jk}^{3}$ epitope of Kidd ( $\mathrm{Jk}$ ) blood groups.

\subsection{Transport studies}

Urea uptake of HUT11 and HUT2 cRNA-injected Xenopus oocytes was 15-50 times faster than in water-injected oocytes depending upon individual experiments, as measured from the initial rate $(90 \mathrm{~s})$. Urea permeabilities $\left(P_{\text {urea }}\right)$ of HUT11- and HUT2-injected oocytes were respectively $14.7 \pm 1.09 \times 10^{-6}$ $\mathrm{cm} / \mathrm{s} \quad(n=13)$ and $45.5 \pm 2.3 \times 10^{-6} \mathrm{~cm} / \mathrm{s} \quad(n=12)$ versus $1.03 \pm 0.18 \times 10^{-6} \mathrm{~cm} / \mathrm{s} \quad(n=10)$ for water-injected oocytes $(P<0.001)$ (Table 1$)$. These values suggest that both HUT2 functions as an efficient urea transporter. It is unknown whether the higher values of permeability for HUT2 cRNAinjected oocytes could be due to a higher number of carrier copies inserted in the membrane rather than to a different activities of each transporter.

The urea flux mediated by HUT2 was inhibited by $0.5 \mathrm{mM}$ phloretin (65\% inhibition), as expected for a urea transporter protein (Table 1). Because pCMBS inhibits water and urea transport by reacting with sulfhydryl groups of the transporters [26,27], the effect of this reagent on HUT2-mediated urea flux was also examined. Surprisingly, we found no inhibition of urea uptake by $0.5 \mathrm{mM}$ pCMBS (Table 1), although this reagent inhibited the erythroid HUT11-mediated urea flux (74\% inhibition). These results are most likely related to the primary structure of HUT11 and HUT2 proteins, particularly regarding the cysteine residues. HUT11 and HUT2 have 10 cysteine residues, among which 7 are conserved and aligned at the same positions. C- $25,-30$ and -151 are present in HUT11 but not in HUT2, whereas C-45, -97 and -288 are present in HUT2 but not in HUT11 (Fig. 1). Based on the predicted membrane topology $[6,8], \mathrm{C}-25$ and $\mathrm{C}-30$ are most likely intracellular, whereas C-151 is located in the 4th transmembrane domain and therefore may represent a likely candidate for pCMBS sensitivity.
In conclusion, we have shown that at least two distinct urea transporters present in human tissues can be distinguished by their functional properties and distribution. The corresponding genes colocalized on chromosome $18 \mathrm{q} 12-\mathrm{q} 21$ and might define a new gene family that evolved by duplication of a common ancestor.

\section{References}

[1] Marsh, D.J. and Knepper, M.A. (1992) Renal handling of urea. In: Renal Physiology (Windhager, E.E., Ed.) pp. 1317-1347. Oxford University Press, Oxford.

[2] Chou, C.-L., Sands, J.M., Nonoguchi, H. and Knepper, M.A. (1990) Am. J. Physiol. 258, 486-494.

[3] Galluci, E., Micelli, S. and Lippe, C. (1975) Nature 255, 722-723.

[4] Macey, R.I. (1984) Am. J. Physiol. 246, C195-C203.

[5] Knepper, M.A. and Star, R.A. (1990) J. Physiol. 259, F393F401.

[6] You, G., Smith, C.P., Kanai, Y., Lee, W.S., Stelzner, M. and Hediger, M.A. (1993) Nature 365, 844-847.

[7] Smith, C.P., Lee, W.-S., Martial, S., Knepper, M.A., You, G., Sands, J.M. and Hediger, M.H. (1995) J. Clin. Invest. 96, 15561563.

[8] Olivès, B., Neau, P., Bailly, P., Hediger, M.A., Rousselet, G., Cartron, J.P. and Ripoche, P. (1994) J. Biol. Chem. 269, 31649-31652.

[9] Smith, C., Shavakul, C., You, G., Martial, S., Lee, W-S., Mackensie, H.S., Sands, J.M., Knepper, M.A. and Hediger, M.A. (1995) American Society of Nephrology, 28th Annual Meeting, November 5-8, San Diego, CA (abstract, p. 329).

[10] Olivès, B., Mattei, M.-G., Huet, M., Neau, P., Martial, S., Cartron, J.-P. and Bailly, P. (1995) J. Biol. Chem. 270, 15607-15610.

[11] Fröhlich, O., Macey, R.I., Edwards-Moulds, J., Gargus, J.J. and Gunn, R.B. (1991) Am. J. Physiol. 260, C778-C783.

[12] Xu, Y., Olivès, B., Bailly, P., Ripoche, P., Ronco, P., Cartron, P.-P. and Rondeau, E. (1995) American Society of Nephrology, 28th Annual Meeting, November 5-8, San Diego, CA (abstract, p. 331).

[13] Pallone, T.L., Work, J., Mayers, R.L. and Jamison, R.L. (1994) J. Clin. Invest. 93, 212-222.

[14] Pallone, T.L. (1994) Am. J. Physiol. 267, R260-R267.

[15] Gillin, A.G. and Sands, J.M. (1993) Semin. Nephrol. 13, 146154.

[16] Mattei, M.G., Philip, N., Passage, E., Moison, J.P., Mandel, J.P. and Mattei, J.F. (1985) Hum. Genet. 69, 268-271.

[17] Laemmli, U.K. (1970) Nature 227, 680-685.

[18] Martial, S., Ripoche, P. and Ibarra, C. (1991) Biochim. Biophys. Acta $1090,86-90$.

[19] Thompson, J.D., Higgins, D.G. and Gibson, T.J. (1994) Nucleic Acids Res. 22, 4673-4680.

[20] Sharp, P.M. and Matassi, G. (1994) Curr. Opin. Genet. Dev. 4, $851-860$.

[21] Bernardi, G. (1993) Mol. Biol. Evol. 10, 186-204. 
[22] Ellsworth, D.L., Hewett-Emmett, D. and Li, W.-H. (1994) Mol. Biol. Evol. 11, 875-885.

[23] Geitvik, G.A., Hoyheim, B., Gedde-Dahl, T., Grzeschilk, K.H., Lothe, R. Tomter, H. and Olaisen, B. (1987) Hum. Genet. 77, 205-209.

[24] Rousselet, G., Bailly, P., and Ripoche, P. (1996) Tandem repeats in urea transporters: identification of an urea transporter signature sequence. Am. J. Physiol. 270, F554-F555.
[25] Promeneur, D., Rousselet, G., Bankir, L., Bailly, P., Cartron, J.P., Ripoche, P., Trinh-Trang and Tran, M.M. (1996) J. Am. Soc. Nephrol. (in press).

[26] Macey, R.I. and Farmer, R.E.L. (1970) Biochim. Biophys. Acta 211, 104-106.

[27] Macey, R.I. (1984) Am. J. Physiol. 246, C195-C203. 